\title{
Asymptomatic hypertransaminasemia in patients in Primary Care
}

\author{
Jonathan Díez-Vallejo ${ }^{1}$ and Ángel Comas-Fuentes ${ }^{1,2}$ \\ ${ }^{1}$ Family and Community Medicine. Centro de Salud Otero. Oviedo, Spain. ${ }^{2}$ Preventive Medicine Public Health \\ Department. University of Oviedo. Spain
}

\begin{abstract}
Introduction and objective: asymptomatic hypertransaminasemia $(\mathrm{AH})$ is a common finding in clinical practice. We propose to determine the prevalence of $\mathrm{AH}$ in our environment, its epidemiology and its evolution.

Material and methods: we studied a random sample of 1,136 blood tests undertaken in 2006, excluding patients with known hepatic disease or symptoms or signs of liver disease, and following the evolution of the AHs until 2009. Data was analyzed using bivariate and multivariate logistic regression.

Results: the percentage of patients found with a $\mathrm{AH}$ was $15.24 \%$ (95\% CI 13.52 to $16.96 \%)$. The rates of AST and ALT, were $6.93 \%$ (95\% CI 5.71 to $8.15 \%$ ) and $14.31 \%$ (95\% CI 12.65 to $15.97 \%$ ) respectively. The percentage of normalization of transaminase values in a second analytical control was a 31.81\% (95\% CI: 26.21 to $37.4 \%)$. An association was found between $\mathrm{AH}$ and BMI (OR: 1.129, 95\% CI 1.062 to 1.199 ) and between the persistence of AH and GGT (OR: 1.011, 95\% CI 1.003 to 1.018). Fundamental tests such as hepatitis serology or ultrasound were performed on only $50 \%$ of patients (with $32 \%$ of steatohepatitis).

Conclusions: the prevalence found in our study of $\mathrm{AH}$ was relatively high (15.24\%), being more frequently encountered in obese subjects. Nearly a third of the AHs is normalized in a subsequent blood test. The high GGT is associated with persistence of AH. There is great variability in terms of additional testing in patients with $\mathrm{AH}$ and the application of such tests is not protocol.
\end{abstract}

Key words: Asymptomatic hypertransaminasemia. Primary care. Liver disease.

Díez-Vallejo J, Comas-Fuentes A. Asymptomatic hypertransaminasemia in patients in Primary Care. Rev Esp Enferm Dig 2011; 103: $530-535$.

Received: 17-12-2010.

Accepted: 08-04-2011.

Correspondence: Ángel Comas. Family and Community Medicine. Centro de Salud Otero. C/ Otero, s/n. 33012 Oviedo, Spain.

e-mail: angel.comas@sespa.princast.es

\section{INTRODUCTION}

Over sixty transamination reactions have been identified in the liver, although the only transaminases with clinical value are the alanine aminotransferase transaminases (ALT) and the aspartate aminotransferase transaminases (AST). Both are used in clinical practice as markers of liver injury, being more specific of liver damage the increase in the parameters of ALT (1). Both are usually present in serum at low concentrations, usually between 3 and $40 \mathrm{U} / \mathrm{L}$, although the normal range varies between different laboratories (2). Its increase above the normal range in patients without symptoms/signs of liver disease is called asymptomatic hypertransaminasemia (AH) (3).

Its prevalence in the general population varies between 4 and $21.7 \%$ (4) between studies and almost a third of people normalize transaminase levels in a second blood control $(5,6)$. This data comes from Anglo Saxon countries that not only have different drug therapies but also have different health systems. There are no recent studies in our country.

The clinical significance of this disease lies in the role of liver disease in the health of the population, being among the leading causes of death (7). The study of AH may contribute to early detection of these diseases.

In recent years, the determination of liver function assessments as part of the routine blood test of patients in primary and special care, and the proliferation of blood tests performed, has led to a significant increase in the detection of alterations of such assessments in the absence of symptoms of liver disease, presenting the clinician with a problem in relation to diagnostic and monitoring (8).

This study has the following objectives: a) to assess the prevalence of $\mathrm{AH}$ in primary care in our country, describing, likewise, the epidemiological characteristics of patients; and b) to determine the time course of transaminase values in subsequent blood tests and follow-up studies performed in these patients. 


\section{METHODS}

We conducted an observational study on patients from the area of the Health Service of Otero (Oviedo), part of the Area IV of the Health Service of the "Principado de Asturias". This is a mixed area (85\% urban) comprising some 26,000 inhabitants.

The study included patients older than 15 years with increased ALT serological parameters and/or AST above normal values and excluded patients with known hepatic disease or symptoms and/or signs of liver disease, and patients outside the area of study. The study period included the years from 2006 through to 2009.

To estimate the prevalence of $\mathrm{AH}$ in a population with an estimated $13 \%$ prevalence, with an error of $2 \%$ and a confidence level of $95 \%$ was necessary to study 1,087 individuals. Patients with an unexpected alteration of transaminases constituted the study sample descriptively and evolutionarily. A sub-sample was selected among patients without alterations in transaminases of size equivalent to comparisons with the previous one.

The following variables were examined by the study: sociodemographic variables (age and sex); anthropometric (body mass index); clinical (presence of hypertension, diabetes, dyslipemia, malignancy, history of transfusion, intravenous drug addiction); habits (alcohol consumption); consumption of hepatotoxic drugs; blood tests (ALT, AST, gamma-glutamyltransferase, alkaline phosphatase, bilirubin, glucose, creatinine, hemoglobin, platelets); temporary (changes in the parameters of transaminases in blood tests); monitoring (prothrombin time, transferrin, thyrotropin, iron, albumin, hepatitis serology, transglutaminase antibodies, abdominal ultrasound, referrals to specialized care); and definitive diagnosis.

We carried out a review involving computerized medical records of patients and a review of blood tests results was made through the laboratory reports. Assuming a minimum of 1,087 blood tests needed (sample size) and an average of 28 tests/day (data obtained through a pre-pilot), it was necessary to review 40 days, which were chosen by simple random method from working days of 2006. The data obtained in those days was distributed as follows: blood tests with elevated transaminases, blood tests with normal transaminases, blood tests in which no parameters were measured and blood tests with increase in transaminases and the presence of some kind of liver disease. With this data, we measured the variables previously mentioned in patients with elevated transaminases. On the other hand, the variables were measured at baseline (not including follow-up variables) in the subgroup of patients with normal transaminases parameters. Data was entered into a database and processed with SPSS for Windows.

We estimated the prevalence of $\mathrm{AH}$ and the proportion of patients who normalized transaminases in subsequent blood tests, with confidence intervals (CI) of $95 \%$. Variables were compared in patients with and without a diagnosis of HA and in patients with and without persistence of HA, by chi-square test for qualitative data and Student $t$ test for quantitative data. Multivariate logistic regression analysis was used to analyze those variables that were associated with the presence and persistence of HA.

\section{RESULTS}

One thousand one hundred and thirty six blood tests were finally studied and data was distributed as follows: 132 blood tests with elevated transaminases; 734 blood tests with normal transaminases; 258 blood tests where transaminases were not measured, 12 blood tests excluded because of the presence of known liver disease.

The percentage of patients with HA was found to be $15.24 \%$ (95\% CI 13.52 to $16.96 \%$ ). In relation to the percentages of elevated AST and ALT, we obtained a $6.93 \%$ (95\% CI 5.71 to $8.15 \%$ ) and $14.31 \%$ (95\% CI: 12.65 to 15 , $97 \%$ ), respectively. Moreover, the percentage found of normalization of transaminases in a second blood test was a $31.81 \%$ (95\% CI: 26.21 to $37.4 \%$ ).

The final sample was 282 patients (132 with AH plus a subsample of 150 randomly obtained from the 734 who did not have it). The average age of the sample was 53.5 years and $56 \%$ were women. The mean values of AST and ALT were 28.2 and $36.3 \mathrm{U} / \mathrm{L}$ respectively. The body mass index (BMI) of the patients was 28.4. 50\% was taking a hepatotoxic drug and $28 \%$ consumed alcohol. These and other characteristics of the sample are summarized in table I, which also makes a comparison of patients with HA compared to those without. The mean values of AST and ALT in patients with $\mathrm{AH}$ was 37.5 and $55.3 \mathrm{U} / \mathrm{L}$ respectively. A notable result is the increased consumption of alcohol in patients with $\mathrm{AH}, 15.9 \%$, compared with $10 \%$ alcohol consumption in patients without $\mathrm{AH}$.

Table II shows the multivariate analysis using as dependent variable the presence of AH with a statistically significantly association with BMI.

Table III shows a comparison of patient characteristics with respect to the persistence or not persistence of elevated transaminases. This highlights the greater proportion of patients with cardiovascular risk factors (hypertension, diabetes, dyslipemia) in patients with persistence of $\mathrm{AH}$. The average value of GGT in patients with AH was approximately twice that of patients without persistence of $\mathrm{AH}$ (80.4 versus $42.1 \mathrm{U} / \mathrm{L}$ ).

Table IV shows the multivariate analysis that uses as the dependent variable the persistence of $\mathrm{AH}$, that was shown to be significantly associated with GGT values.

Figure 1 shows the proportion of patients in whom there is normalization of transaminases during the period 20062009 , confirming the growing trend of normalization of liver function tests during the study period.

\section{DISCUSSION}

The prevalence found in our study for AH was $15.24 \%$. This figure is within that contained in the bibliography. 
Table I. Description of the characteristics of the sample studied and compared between patients with or without asymptomatic hypertransaminasemia

\begin{tabular}{|c|c|c|c|c|}
\hline Variables & Total & $A H$ yes & AH no & "p" \\
\hline Age & $53.5(17.3)$ & $53.2(15.3)$ & $53.8(18.9)$ & 0.763 \\
\hline Gender & & & & 0.017 \\
\hline Male & $44.0(124)$ & $51.5(68)$ & $37.3(56)$ & \\
\hline Female & $56.0(158)$ & $48.5(64)$ & $62.7(94)$ & \\
\hline $\begin{array}{l}\text { High blood } \\
\text { pressure }\end{array}$ & & & & 0.434 \\
\hline Yes & $26.6(75)$ & $28.8(38)$ & $24.7(37)$ & \\
\hline No & $73.4(207)$ & $71.2(94)$ & $75.3(113)$ & \\
\hline Diabetes & & & & 0.146 \\
\hline Yes & $14.9(42)$ & $18.2(24)$ & $12.0(88)$ & \\
\hline No & $85.1(240)$ & $81.8(108)$ & $88.0(132)$ & \\
\hline Dyslipidemia & & & & 0.083 \\
\hline Yes & $28.4(80)$ & $33.3(44)$ & $24.0(36)$ & \\
\hline No & $71.6(202)$ & $66.7(88)$ & $76.0(114)$ & \\
\hline Alcohol & & & & 0.064 \\
\hline Yes & $12.8(36)$ & $15.9(21)$ & $10.0(15)$ & \\
\hline No & $87.2(246)$ & $84.1(111)$ & $90.0(135)$ & \\
\hline Hepatotoxic drugs & & & & 0.002 \\
\hline Primary & $22.7(64)$ & $27.3(36)$ & $18.7(28)$ & \\
\hline Secondary & $27.3(77)$ & $17.4(23)$ & $36.0(54)$ & \\
\hline No & $50.0(141)$ & $55.3(73)$ & $45.3(68)$ & \\
\hline $\begin{array}{l}\text { Body mass index } \\
\text { (BMI) }\end{array}$ & $28.4(5.0)$ & $29.8(4.9)$ & $26.9(5.2)$ & $<0.001$ \\
\hline AST (U/L) & $28.2(17.2)$ & $37.5(21.3)$ & $20.0(5.8)$ & $<0.001$ \\
\hline $\operatorname{ALT}(\mathrm{U} / \mathrm{L})$ & $36.3(33.0)$ & $55.3(40.2)$ & $19.7(5.8)$ & $<0.001$ \\
\hline Gamma-GT (U/L) & $44.2(60.3)$ & $71.1(78.4)$ & $20.9(16.5)$ & $<0.001$ \\
\hline Bilirubin (mg/dl) & $0.7(0.6)$ & $0.7(0.4)$ & $0.6(0.8)$ & 0.032 \\
\hline $\begin{array}{l}\text { Alkaline phosphatase } \\
(\mathrm{U} / \mathrm{L})\end{array}$ & $78.2(42.4)$ & $86.2(55.4)$ & $70.3(20.1)$ & 0.002 \\
\hline Glucose (mg/dl) & $100.4(31.1)$ & $104.6(35.7)$ & $96.7(26.1)$ & 0.034 \\
\hline Creatinine (mg/dl) & $0.9(0.2)$ & $0.9(0.2)$ & $0.8(0.2)$ & 0.164 \\
\hline Haemoglobin (g/dl) & $14.4(1.3)$ & $14.7(1.3)$ & $14.1(1.2)$ & $<0.001$ \\
\hline Platelets (mil/ $\mu \mathrm{g})$ & $249.6(62.0)$ & $247.5(52.9)$ & $251.4(69.1)$ & 0.597 \\
\hline
\end{tabular}

In the qualitative data percentage is shown and, in brackets, absolute numbers. In the quantitative data the mean is shown and, in brackets, the standard deviation. $\mathrm{AH}$ : asymptomatic hypertransaminasemia.
While studies such as that conducted in the U.S. found a prevalence of $8.1 \%$ (9), others like the one published in Scotland get a $21.7 \%$ (10) of prevalence. Other authors report that the prevalence of hypertension in the general population ranges between $4-13 \%$ (4). These differences may be explained by several reasons: because the prevalence of hypertension varies with the type of population studied and the variability in different laboratories in the normal range of transaminases (11).

On the other hand, the prevalence of isolated rise of AST and ALT was 6.93 and $14.31 \%$, respectively, having also differences with the previously mentioned studies for the same reasons already stated regarding the prevalence of hypertension. It is striking that the prevalence of increased ALT is similar to that of AH, being, meanwhile, about twice that of AST (14.21 versus 6.93). This fact is mainly explained by the higher concentration of ALT in the liver, and its specific rise of liver damage.

The mean values of AST and ALT in patients with AH were $37.5 \pm 21.3 \mathrm{U} / \mathrm{L}$ and $55.3 \pm 40.2 \mathrm{U} / \mathrm{L}$, respectively, similar to those found in other studies, such as Khedmat et al. in 2007 (12). This confirms that the majority of increases above normal values for transaminases are mild, often below twice the normal range. Several studies claim that $\mathrm{AH}$ is associated with an increased risk of developing cardiovascular disease, hypertension, diabetes and dyslipidemia (12). In our study, despite significant percentage of patients with these diseases among those with $\mathrm{AH}$ (28.8\% of hypertensives, diabetics $18.2 \%$ and $33.3 \%$ of patients with dyslipidemia), no association was found statistically significant among these factors and the presence or persistence of $\mathrm{AH}$, after carrying out a multivariate analysis controlling other variables. In turn, we found a relationship between elevated GGT and the persistence of AH. This finding could be explained by the close relationship between GGT levels and the presence of metabolic syndrome, as demonstrated by previous studies (13), but also by the fact that alteration of this enzyme is a marker of increased liver damage.

Alcohol consumption is associated with liver damage and the development of advanced liver disease. The per-

Table II. Multivariate logistic regression analysis using

as dependent variable the presence of asymptomatic hypertransaminasemia $(0=$ no $\mathrm{AH})$

\begin{tabular}{llllll}
\hline & Coefficient & D.S. & " $p$ " & OR & (95\% IC for OR) \\
\hline Age & -0.017 & 0.009 & 0.056 & 0.983 & $(0.966-1.000)$ \\
Gender (0 = Female) & 0.468 & 0.281 & 0.096 & 1.597 & $(0.920-2.773)$ \\
Hypertension (0 =No) & -0.182 & 0.340 & 0.592 & 0.834 & $(0.428-1.623)$ \\
Diabetes (0 = No) & 0.384 & 0.389 & 0.324 & 1.468 & $(0.685-3.145)$ \\
Dyslipedemia (0 = No) & 0.230 & 0.381 & 0.547 & 1.258 & $(0.596-2.653)$ \\
Alcohol (0 = No) & 0.377 & 0.432 & 0.384 & 1.457 & $(0.624-3.401)$ \\
Medication (0 = No) & 0.484 & 0.409 & 0.237 & 1.623 & $(0.727-3.621)$ \\
BMl & 0.121 & 0.031 & $<0.001$ & 1.129 & $(1.062-1.199)$ \\
Constant & -3.130 & 0.908 & 0.001 & 0.044 & \\
\hline
\end{tabular}

AH: asymptomatic hypertransaminasemia; Alcohol: alcohol intake; Medication: primary hepatotoxic drugs intake; BMI: body mass index. 
centage of patients who drink with $\mathrm{AH}$ was $15.9 \%$ compared with $10 \%$ who did not have it, this was significant at bivariate level $(\mathrm{p}=0.064)$ although it was not significant in the multivariate analysis. As for the persistence of $\mathrm{AH}$ the percentage of drinkers was higher in those with the alteration (25 vs. 15.6\%) but showed no statistical association despite the known relationship between liver damage and alcohol consumption.

Medication-induced liver toxicity should always be considered faced with a rise of transaminases parameters, although there is wide clinical and analytical variability. There are no consistent patterns of incidence and prevalence of hepatic adverse effects of medication, and clinical significance of hepatotoxicity is highly variable (14). This study found $44.7 \%$ of $\mathrm{AH}$ patients who had used potentially hepatotoxic drugs, and among them, $27.3 \%$ had used hepatotoxic drugs that we have called primary, mainly statins and methotrexate. Significant difference at the bivariate level, but not confirmed by controlling other parameters in the multivariate analysis. The same thing happened between the act of using these drugs and the persistence of $\mathrm{AH}$.

In recent years, several studies have shown that elevated liver enzymes are often found in people with obesity (15), as measured by BMI. We found that the average BMI of patients with HA was 29.8, similar to that obtained in other studies of BMI levels around 27-30 (12). Furthermore, in the multivariate analysis in our study we observed a statistically significant association between the patient's BMI and the presence of $\mathrm{AH}$, an increase of $12.5 \%$ in the probability of $\mathrm{AH}$ for each point increase in BMI. Moreover, the average $\mathrm{BMI}$ of patients with persistence of $\mathrm{AH}$ was 30.1 , similar to that of patients with $\mathrm{AH}$, not being, therefore, statistically significant association between BMI and the persistence of $\mathrm{AH}$, after a multivariate analysis controlling the other variables was carried out.

In relation to gender, the proportion of men and women with $\mathrm{AH}$ was around $50 \%$, with an almost significant influence bivariate level ( $p=0.096$ ) but not significant in the multivariate analysis. Perhaps in the future one should consider the convenience of adjusting the range of normal
Table III. Comparison between patients with or without asymptomatic hypertransaminasemia

\begin{tabular}{|c|c|c|c|}
\hline \multirow{2}{*}{$\begin{array}{l}\text { Variables } \\
\text { Age }\end{array}$} & \multirow{2}{*}{$\begin{array}{l}\text { AH persists } \\
54.3(13.4)\end{array}$} & \multicolumn{2}{|c|}{ AH no persists " $p$ " } \\
\hline & & $49.6(20.1)$ & 0.125 \\
\hline Gender & & & 0.008 \\
\hline Male & $58.0(58)$ & $31.3(10)$ & \\
\hline Female & $42.0(42)$ & $68.8(22)$ & \\
\hline Hypertension & & & 0.587 \\
\hline Yes & $30.0(30)$ & $25.0(8)$ & \\
\hline No & $70.0(70)$ & $75.0(24)$ & \\
\hline Diabetes & & & 0.138 \\
\hline Yes & $21.0(21)$ & $9.4(3)$ & \\
\hline No & $79.0(79)$ & $96.6(29)$ & \\
\hline Dyslipidemia & & & 0.044 \\
\hline Yes & $38.0(38)$ & $18.8(6)$ & \\
\hline No & $62.0(62)$ & $81.3(26)$ & \\
\hline Alcohol & & & 0.271 \\
\hline Yes & $25.0(25)$ & $15.6(5)$ & \\
\hline No & $75.0(75)$ & $84.4(27)$ & \\
\hline Hepatotoxic drugs & & & 0.365 \\
\hline Primary & $30.0(30)$ & $18.8(6)$ & \\
\hline Secondary & $18.0(18)$ & $15.6(5)$ & \\
\hline No & $52.0(52)$ & $65.6(21)$ & \\
\hline Body mass index & $30.1(5.1)$ & $28.8(3.9)$ & 0.230 \\
\hline AST (U/L) & $39.0(23.0)$ & $44.3(11.4)$ & 0.160 \\
\hline $\operatorname{ALT}(\mathrm{U} / \mathrm{L})$ & $58.8(45.3)$ & $19.7(5.8)$ & 0.075 \\
\hline Gamma-GT (U/L) & $80.4(79.8)$ & $42.1(67.4)$ & 0.016 \\
\hline Bilirubin (mg/dl) & $0.7(0.4)$ & $0.7(0.3)$ & 0.609 \\
\hline Alkaline phosphatase (U/L) & $88.0(62.0)$ & $80.5(27.2)$ & 0.514 \\
\hline Glucose (mg/dl) & $107.1(39.2)$ & $96.7(19.7)$ & 0.151 \\
\hline Creatinine (mg/dl) & $0.9(0.2)$ & $0.8(0.2)$ & 0.210 \\
\hline Hemoglobin (g/dl) & $14.9(1.3)$ & $14.1(1.3)$ & 0.006 \\
\hline Platelets (mil/ug) & $272.5(53.9)$ & $239.5(50.2)$ & 0.002 \\
\hline
\end{tabular}

In the qualitative data the percentage is shown and, in brackets, absolute numbers In the quantitative data the mean is shown and, in brackets, the standard deviation. $\mathrm{AH}$ : asymptomatic hypertransaminasemia.

Table IV. Multivariate logistic regression analysis using as dependent variable persistence of asymptomatic hypertransaminasemia $(0=$ no persistent $\mathrm{AH})$

\begin{tabular}{|c|c|c|c|c|c|}
\hline & Coefficient & D.S. & "p" & $O R$ & (95\% IC for OR) \\
\hline Age & -0.014 & 0.015 & 0.358 & 0.986 & $(0.957-1.016)$ \\
\hline $\mathrm{BMI}$ & 0.070 & 0.042 & 0.096 & 1.072 & $(0.988-1.164)$ \\
\hline Alcohol $(0=$ No) & 0.017 & 0.517 & 0.973 & 1.017 & $(0.369-2.804)$ \\
\hline Medication $(0=\mathrm{No})$ & -0.048 & 0.476 & 0.920 & 0.953 & $(0.375-2.423)$ \\
\hline GGT & 0.011 & 0.004 & 0.005 & 1.011 & $(1.003-1.018)$ \\
\hline Constant & -2.111 & 1.508 & 0.161 & 0.121 & \\
\hline
\end{tabular}

AH: asymptomatic hypertransaminasemia; Alcohol: alcohol intake; Medication: primary hepatotoxic drugs intake; BMI: body mass index. 


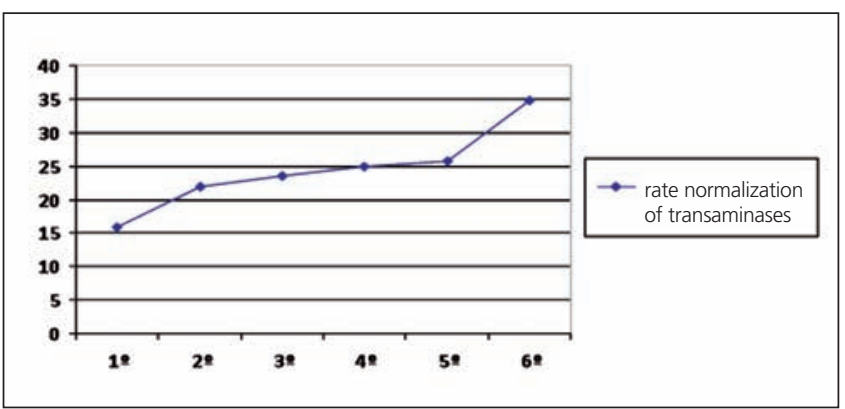

Fig. 1. Evolution of the percentage of normalization of transaminases be tween 2006-2009 per semester.

transaminases gender and BMI as suggested by some authors (16), although they are population studies without histological confirmation.

The rise above the normal range of GGT is often associated with the development of liver disease; that is why studies like the one published in Scotland (9) recommend the systematic determination of GGT in the request for additional tests conducted in Primary Care. The mean value of GGT in patients with persistence of $\mathrm{AH}$ was $71.1 \mathrm{U} / \mathrm{L}$, and the percentage of high values of GGT in these patients was $88.4 \%$. Therefore, it has been demonstrated an association between increased GGT and the persistence of AH, by multivariate analysis, finding a $1.1 \%$ increase in the probability of persisting AH for each unit of increase in GGT. It is striking that while there is this association, the persistence of HA was not significantly associated with a greater increase in the number of transaminases.

Different studies have shown a high percentage of patients with $\mathrm{AH}$ in which the transaminases values are normalised in subsequent blood tests (8) Despite this, few physicians repeat liver function when faced with a patient with $\mathrm{AH}$. In our study, the percentage of normalization of the serum levels of transaminases was $31.81 \%$, similar to the percentage published in the previously mentioned studies. During the following three years (2006-2009) it was confirmed the progressive normalization of transaminases over time, as shown by other studies. Therefore, it confirms the relevance of repeating the levels of transaminases in dealing with all patients with $\mathrm{AH}$ before initiating further studies more expensive or complex.

In all patients with increased serum levels of transaminases, after a thorough history and physical examination, a battery of additional staggered tests is recommended if the increased levels persist (17). There is no consensus or single diagnostic algorithm for conducting such tests, leaving the decision to the discretion of the physician. In our study, only hepatitis serology was requested in $50 \%$ of cases, all being negative. It is also striking that all patients with continued $\mathrm{AH}$, an abdominal ultrasound was carried out in only $50 \%$, with about one third being normal and two thirds showing fatty liver. It is surprising the low percentage of relevant additional tests requested from patients with per- sistence of AH. Therefore, it would be essential to promote and publicize the importance of a proper study of patients with $\mathrm{AH}$ in order to make possible an early diagnosis of liver disease, and also create unified algorithms of performance for a better approach to this kind of alteration. However, most tests carried out were normal, not arriving to a final diagnosis different to hepatic steatosis, except for a case of hemochromatosis and another of hepatic porphyra.

As for the limitations of our study, various aspects should be highlighted. One of the most important is the lack of data for some of the variables, which can influence reporting if the fact of having more disease, as an unexplained alteration of transaminases, determines that the histories of these patients are more comprehensive than those of comparatively healthy ones.

On the other hand, the lack of association between cardiovascular disease and alcohol consumption and the presence of AH may be due to the limited size of the sample, as there are studies in the literature that do establish the relationship.

In conclusion, the prevalence found in our study of $\mathrm{AH}$ was relatively high (15.24\%) and those subjects with $\mathrm{AH}$ were more frequently obese. Nearly a third is normalized in a subsequent blood test. The high GGT is associated with persistence of $\mathrm{AH}$. There is great variability in terms of additional testing in patients with $\mathrm{AH}$, and the application of such tests is not protocol.

\section{REFERENCES}

1. Dufour DR, Lott JA, Nolte FS, Gretch DR, Koff RS, Seeff LB. Diagnosis and monitoring of hepatic injury. Recommendations for use of laboratory tests in screening, diagnosis and monitoring. Clin Chem 2000;46:2050-68.

2. Servicio de Bioquímica clínica [sede web]. Oviedo: Hospital Universitario Central de Asturias; 2010 [acceso 21 de junio de 2010]. Valores normales de análisis clínicos. Disponible en: www.hca.es

3. Álvarez-Martínez H, Pérez E. El paciente con hipertransaminasemia. [monografía en internet]. Oxaca, México. Rev Fac Med UNAM. 2005. [acceso 23 de Junio de 2010]. Disponible en: http://www.ejournal.mx/ rfm/no48-2/RFM48205.pdf

4. Reggiardo V. Hipertransaminasemia asintomática. [monografía en Internet]. Buenos Aires: Servicio de Gastroenterología y Hepatología del Hospital Centenario; 2008 [acceso 19 de mayo de 2010]. Disponible en: http://www.aaeeh.org.ar/rco/2008/segunda/ResumenDraReggiardo.pdf

5. Green R, Flamm S. AGA technical review on the evaluation of liver chemistry tests. Gastroenterology 2002;123:1367-84.

6. Lazo M, Selvin E, Clark J. Brief communication: clinical implications of short-term variability in liver test results. Ann Inter Med 2008; 148:348-52.

7. Ministerio de Sanidad y Consumo [sede web]. Instituto de Información Sanitaria: Mortalidad por causas de muerte: España y comunidades autónomas; 2008 [acceso 15 de marzo de 2010]. Disponible en: http://www.msps.es/estadEstudios/estadisticas/estadisticas/estMinisterio/mortalidad/mortalidad.htm

8. Cuadrado A, Crespo J. Hipertransaminasemia en pacientes con negatividad de marcadores virales. Rev Esp Enferm Dig 2004;96:484500 .

9. Ioannou G, Boyko E, Lee S. The prevalence and predictors of elevated serum aminotransferase activity in the United States in 1999-2002. Am J Gastroenterol 2006;101:76-82.

10. Donnan PT, McLernon D, Dillon JF, Ryder S, Roderick P, Sullivan F, et al. Development of a decision support tool for primary care mana- 
gement of patients with abnormal liver function tests without clinically apparent liver disease: a record-linkage population cohort study and decision analysis (ALFIE). Health Technol Assess 2009;13:3-93.

11. Daniel S, Pratt MD, Marshall M, Kaplan MD. Evaluation of abnormal liver-enzyme results in asymptomatic patients. N Eng J Med 2000;342: 1266-71.

12. Khedmat H, Fallahian F, Abolghasemi H, Hajibeigi B, Attarchi Z, Alaeddini F, et al. Serum gamma-glutamyltransferase, alanine aminotransferase, and aspartate aminotransferase activity in Irian healthy blood donor men. World J Gastroenterol 2007;13:889-94.

13. Marchesini G, Avagnina S, Barantani EG, Ciccarone AM, Corica F, Dall'Aglio E, et al. Aminotransferase and gamma-glutamyltranspeptidase levels in obesity are associated with insulin resistance and metabolic syndrome. J Endocrinol Invest 2005;28:333-9.
14. García-Cortés M, Andrade RJ, Lucena MI, González-Grande R, Camargo R, Fernández Bonilla E, et al. Hepatotoxicidad secundaria a fármacos de uso común. Gastroenterol Hepatol 2005;28:461-72.

15. Weon Choi J. Association between elevated serum hepatic enzyme activity and total body fat in obese humans. Ann Clin Lab Sci 2003; 33:257-64.

16. Lott JA, Nolte FS, Gretch DR, Koff RS, Seeff LB. Laboratory guidelines for screening, diagnosis and monitoring of hepatic injury. National Academy of Clinical Biochemistry 2000;12:4-11.

17. Fisterra.com, Atención Primaria en la Red [sede web]. Lugo: Fisterra.com; Junio 2009 [acceso el 23 de Junio de 2010]. Díaz Otero A, de la Fuente Sánchez S, Castiñeira Pérez C, Costeribas C. Hipertransaminasemia. Guías Clínicas Fisterra; [aproximadamente 3 pantallas]. Disponible en: http://www.fisterra.com/guias2/transaminasas.asp 